\title{
Deployment of a Mobile Laboratory for the Control and Monitoring of High- Consequence Infectious Diseases: An Illustration With the Ebola Virus, the Biowarfare Agents, and the COVID-19
}

\author{
Omar Nyabi \\ Université Catholique de Louvain (UCL) \\ Olga Vybornova \\ Université Catholique de Louvain (UCL) \\ Jean-Luc Gala \\ Université Catholique de Louvain (UCL)
}

\begin{abstract}
The novel coronavirus (COVID-19) pandemic has caused societal issues, economic and political tensions worldwide. This shows, once more, that dissemination of correct information based on scientific evidence together with a quick and concerted action is the key to build a sound capability for the management of biological emergencies.
\end{abstract}

Here, we summarize the lessons learned from our preparedness and intervention during (i) our deployment during the 2014-2016 Ebola outbreak in West Africa; (ii) our large-scale exercises from Horizon 2020 Security program where the focus is on handling intentional dispersion of infectious agents; and (iii) our fight against COVID-19: by the deployment of Biological Light Fieldable Laboratory for Emergencies (BLiFE mobile laboratory) in Turin and Novara, Piedmont Region, Italy.

At the latter deployment, the ultimate goal was a large screening for COVID-19 prevalence in primo intervention individuals. It cannot be ignored that the COVID-19 pandemic is an ideal situation to whet our preparedness, coordination of response and risks monitoring in case of future biological threats or attacks.

Keywords: Biological Light Fieldable Laboratory for Emergencies (B-LiFE), biological threats, pandemic response, global health risks, deployment in remote locations, laboratory information management system (LIMS)

\section{INTRODUCTION}

The Center for Applied Molecular Technologies (CTMA) platform developed and directed by Prof, $\mathrm{PhD}$ and MD Gala JL, has a special constitution comprised of three components: military, clinical, and academic. The platform is the cutting-edge biotechnology center whose main mission, in its military 
component, is the rapid identification and control of biological threats regardless of their origin: natural, accidental, or intentional.

In return, this specific activity has proven to be extremely beneficial for many patients that are affected by pathogens for which diagnosis was difficult or even impossible at University Hospital Saint-Luc (CUSL). Moreover, being affiliated to the Institute for Experimental and Clinical Research (IREC) is also beneficial because of the possibility to be associated with a large number of targeted research projects at both national and international levels. The defense division also has a highly experienced multidisciplinary university research team, and the university has a team of military researchers specialized in the field of infectious diseases and trained to deal with the challenges of rapid deployment during major health crises. As for the University Hospital Saint Luc, the Defense division benefits also from its innovative and highperformance methodological developments.

\section{GENERAL SETUP IN THE B-LIFE MOBILE LABORATORY}

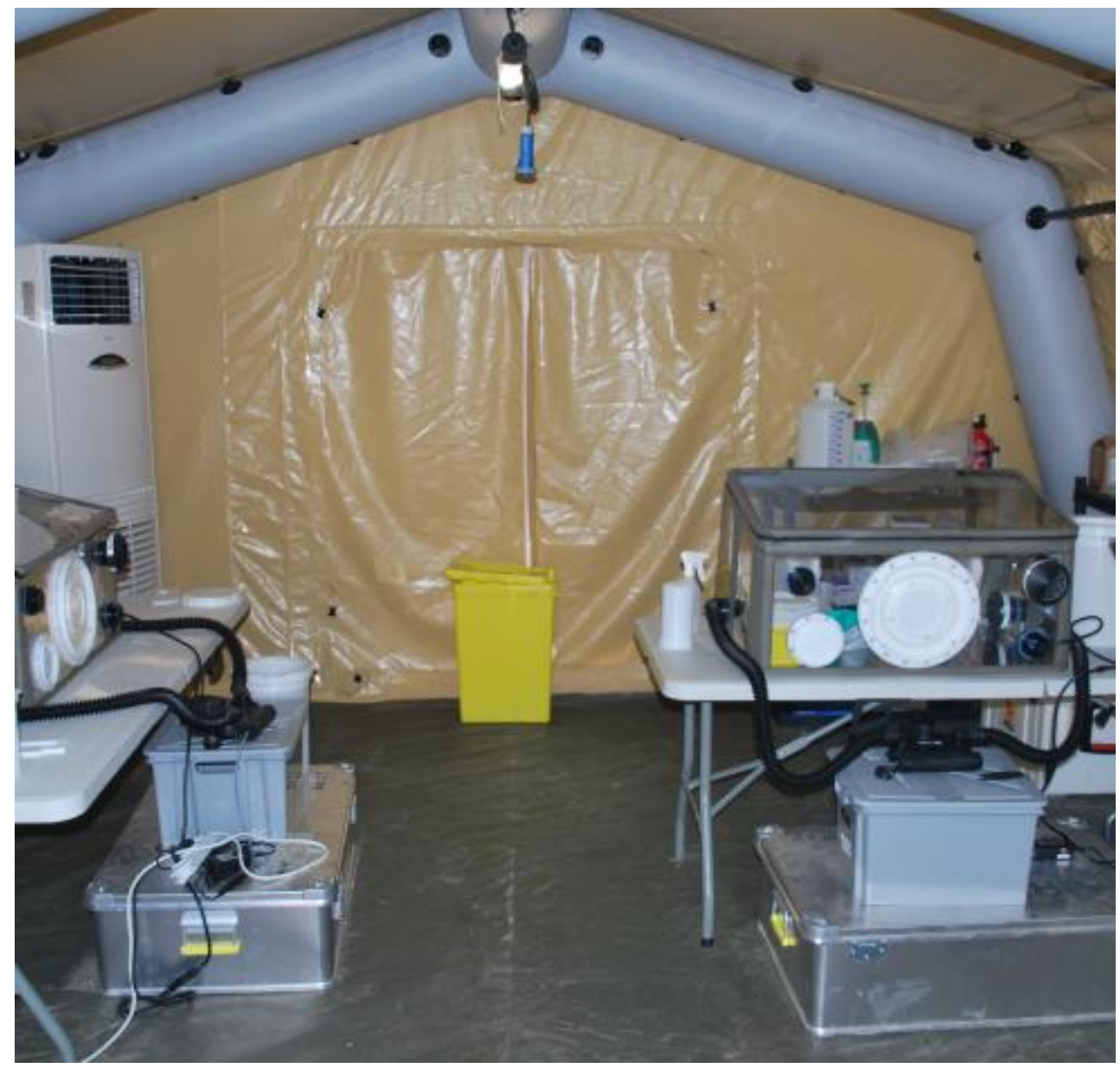

\section{Deployment in West Africa During Ebola Pandemics}

The development of a mobile laboratory by CTMA has proved its worth in the field, especially during deployment in Guinea between December 2014 and March 2015. This analytical deployable capacity called Biological Light Fieldable Laboratory for Emergencies (B-LiFE), is light, easily transportable, and deployable to the remotest corners of the world. Its aim is to rapidly identify deadly infectious agents to facilitate their control. This has enabled us to contribute to international efforts to combat the Ebola epidemic in West Africa. As a result of the composite structure of CTMA, we were one of the few teams 
comprising members from diverse fields like academic, clinical, and military divisions to get deployed. The laboratory personnel are well-trained for work and managing biosafety constraints of a field laboratory. Despite total isolation during our deployment, in the forest area of N'Zerekore in Guinea, we were clearly one of the teams that functioned best on the ground.

\section{FIGURE 2 \\ DEPLOYMENT CAMP IN GUINEA, WEST AFRICA; DECEMBER 2014 - MARCH 2015}

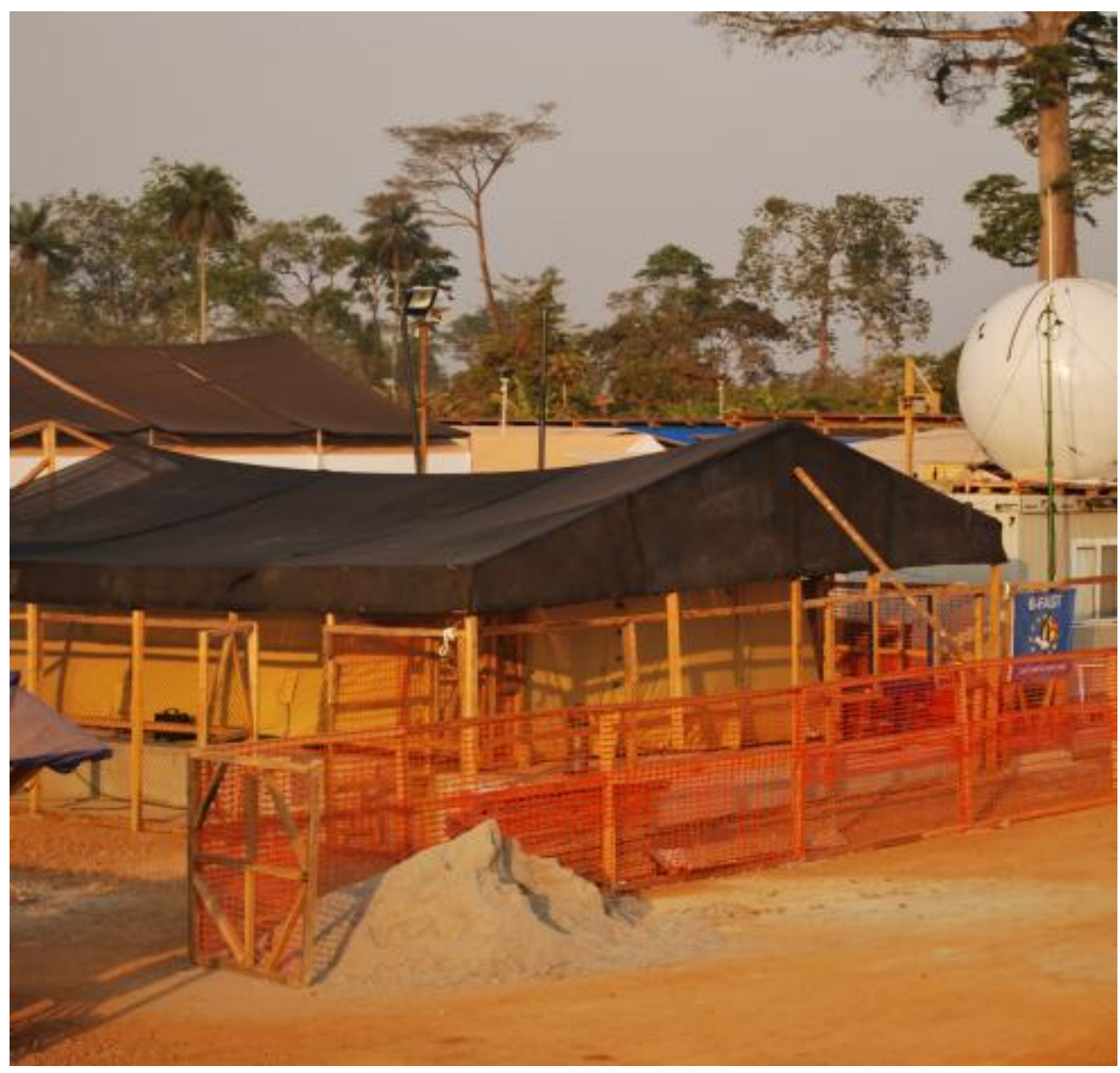

However, this laboratory does not remain inactive when Africa is not in the grip of any epidemic. Generally, between the missions it serves as a technological incubator. We evaluate and develop a series of emerging technologies, which we adapt to the context on the ground, in collaboration with Belgian and other European companies. Once the techniques have been developed and validated, we foster their implementation wherever needed. This expertise has enabled us to develop an infectious disease surveillance laboratory in Bukavu in the Democratic Republic of Congo among our other activities. In this case, as in others, we attempted to develop the most appropriate, rapid, and affordable diagnostic methods.

\section{Bioterrorism: A Full-Scale Test Exercise in Europe}

CTMA had launched B-LiFE in a full-scale scenario, in 2016 and for the first time, two light fieldable laboratories were deployed side by side in Munich, Germany: B-LiFE and the laboratory of the Bundeswehr Institute for Microbiology. This novel exercise reproduced a scenario where "Criminal dispersion of an infectious agent" has occurred; the aim was to evaluate and compare the results of each laboratory and to test their performance and interoperability. The pathogens (viruses and bacteria) infecting biological 
samples were, of course, harmless simulants, but the methods and equipment were actually those used in real-life crisis or epidemic situations. We also utilized the state-of-the-art technology for geo-locating samples by means of telecommunication solutions - satellite communications combined with terrestrial communications that provided a good and stable internet connection. In addition, we evaluated for the first time the MinION third generation sequencing (NGS) for identifying infectious agents in the field. High troughput sequencing on the field is indeed a vital method for rapid monitoring and detection of known and unknown epidemic pathogens (Yadav et al., 2020).

\section{Deployment in Italy During COVID-19 Pandemic}

Recently, from June 10, 2020, to July 23, 2020, the B-LiFE mobile laboratory of CTMA, under the guidance of Prof. Jean-Luc Gala traveled to Italian Piedmont to assist the Italian authorities in their fight against the COVID-19 pandemic. This mission was supported by the European Space Agency (ESA Contract No. 4000131106/20/NL/AS issued 2020/04/06) which made available a budget for this six-week deployment in two different locations (Turin and Novara). During this mission, the mobile laboratory tested more than 6,000 front-line healthcare workers (Croce Rossa Italiana), volunteers from the civil protection services (Civile Protezione Italiana), and the police (Carabinieri) to assess their past and current exposure to the virus. The results provided real-time information on the exposure of these first responders to severe acute respiratory syndrome coronavirus 2 (SARS-CoV-2), the virus responsible for COVID-19, during the fight against the pandemic. Based on our preliminary results, it was observed that the first responders in Novara, a region close to Lombardy heavily affected by the disease, were more exposed than those working in the more remote city of Turin. The rate of positive antibodies was twice as high among the first responders in Novara than in Turin. The first responders from Croce Rossa Italiana were also found to be clearly more exposed to the coronavirus than those from Protezione Civile Italiana.

FIGURE 3

\section{DEPLOYMENT IN PIEMOND, ITALY, DURING COVID-19 PANDEMIC. THE WHOLE TEAM FROM ITALY, FRANCE AND BELGIUM WERE PRESENT}
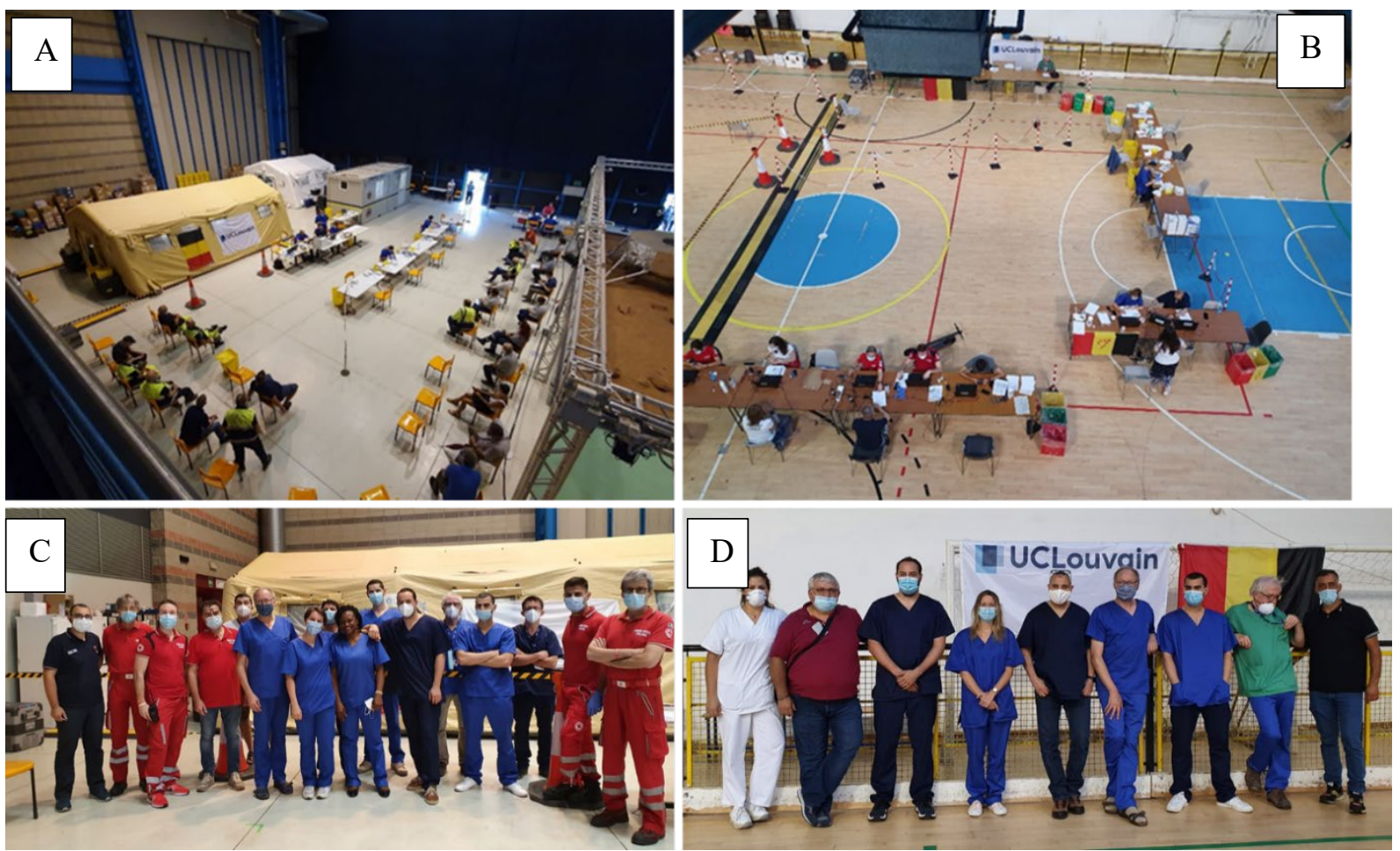

A: Turin; B: staff operating in Turin; C: Novara and D: Staff operating in Novara 
The mission also successfully confirmed the feasibility of applying key new operational strategies that determine the success or failure of such a rapid response in the context of a major acute crisis that could seriously affect a region or country inside or outside the European Union. The concept of "mass screening of citizens" by a deployed mobile laboratory and the requirements for effective interconnectivity of the patients' database results generated by the laboratory with the host country's national IT e-health platform is a key pillar in rapid crisis management. The mobile laboratory data is stored, managed and transmitted by means of a Laboratory Information Management System (LIMS) (Paul \& Chatterjee, 2020).

The concept of scalability and interoperability was also brilliantly demonstrated by the very successful collaboration of French scientists from the Pasteur Institute, Paris and Italian scientists from the University of Turin. All were invited to join the B-LiFE team after a quick hands-on training in the field. This allowed us to maximize the work of the scarce-staff resources during the entire duration of this exhausting mission, and to successfully re-locate the laboratory from Turin to Novara in the last part of the mission with the same operational efficiency. This mission also demonstrated the crucial role of the new telecommunication protocols developed by B-LiFE, which effectively combined terrestrial and satellite communications in a new emergency telecommunications node (TEN). It should be noted that a new generation SatCom antenna was generously provided to B-LiFE for the entire mission management by the Luxembourg Minister of Defense and that the French SMEs ETELM (who managed telecommunication optimization) and AgileBio (who managed adaptation of LIMS to specific operational conditions) (de Pelsemaeker, Guiot, Vanderveken, Galant, \& Van Bockstal, 2020; Weemaes et al., 2020) provided unrestricted support throughout the mission. Finally, this mission was also a perfect showcase for the tests produced in Belgium and used to detect SARS-CoV-2 antibodies and viral signatures in a mobile laboratory.

In conclusion, this B-LiFE mission unequivocally demonstrated the spirit of the European unity that encouraged all team members, including those from countries supporting the B-LiFE team, making this challenging operation a complete success in medical, scientific and humanitarian aspects. Furthermore, a rapid response mobile laboratory, which is flexible and adaptable with a very narrowed technological watch when associated with LIMS is the starting point:

- for facing emerging biological threats, including those related to climate change, food safety, security, and bioterrorism (Irenge \& Gala, 2012) and their social and economic impact on public health:

- for rapid detection/identification of biological agents and bio forensics using innovative, fast, and effective methods (Bentahir, Ambroise, Delcorps, Pilo, \& Gala, 2018);

- $\quad$ for catering to needs, gaps, and proposals to increase awareness and preparedness concerning responses to newborn biological emergencies of public health organizations.

\section{REFERENCES}

Bentahir, M., Ambroise, J., Delcorps, C., Pilo, P., \& Gala, J.L. (2018). Sensitive and Specific Recombinase Polymerase Amplification Assays for Fast Screening, Detection, and Identification of Bacillus anthracis in a Field Setting. Appl Environ Microbiol, 84(11). doi:10.1128/AEM.00506-18

de Pelsemaeker, M.C., Guiot, Y., Vanderveken, J., Galant, C., \& Van Bockstal, M.R. (2020). The Impact of the COVID-19 Pandemic and the Associated Belgian Governmental Measures on Cancer Screening, Surgical Pathology and Cytopathology. Pathobiology, pp. 1-10. doi:10.1159/000509546

Irenge, L.M., \& Gala, J.L. (2012). Rapid detection methods for Bacillus anthracis in environmental samples: A review. Appl. Microbiol. Biotechnol., 93(4), 1411-1422. doi:10.1007/s00253-011$3845-7$

Paul, S., \& Chatterjee, M.K. (2020). Data Sharing Solutions for Biobanks for the COVID-19 Pandemic. Biopreserv Biobank. doi:10.1089/bio.2020.0040

Weemaes, M., Martens, S., Cuypers, L., Van Elslande, J., Hoet, K., Welkenhuysen, J., . . Goveia, J. (2020). Laboratory information system requirements to manage the COVID-19 pandemic: A 
report from the Belgian national reference testing center. J Am Med Inform Assoc, 27(8), 12931299. doi:10.1093/jamia/ocaa081

Yadav, P.D., Shete-Aich, A., Nyayanit, D.A., Pardeshi, P., Majumdar, T., Balasubramanian, R., .. . Mourya, D.T. (2020). Detection of coronaviruses in Pteropus \& Rousettus species of bats from different States of India. Indian J. Med. Res., 151(2\&3), 226-235. doi:10.4103/ijmr.IJMR_795_20 